PROCEEDINGS OF THE

AMERICAN MATHEMATICAL SOCIETY

Volume 128, Number 10, Pages 2921-2931

S 0002-9939(00)05324-7

Article electronically published on March 2, 2000

\title{
A NONTRIVIAL EXAMPLE OF APPLICATION OF THE NIELSEN FIXED-POINT THEORY TO DIFFERENTIAL SYSTEMS: PROBLEM OF JEAN LERAY
}

\author{
JAN ANDRES
}

(Communicated by Dale Alspach)

\begin{abstract}
In reply to a problem posed by Jean Leray in 1950, a nontrivial example of application of the Nielsen fixed-point theory to differential systems is given. So the existence of two entirely bounded solutions or three periodic (harmonic) solutions of a planar system of ODEs is proved by means of the Nielsen number. Subsequently, in view of T. Matsuoka's results in Invent. Math. (70 (1983), 319-340) and Japan J. Appl. Math. (1 (1984), no. 2, 417434), infinitely many subharmonics can be generically deduced for a smooth system. Unlike in other papers on this topic, no parameters are involved and no simple alternative approach can be used for the same goal.
\end{abstract}

\section{Historical Remarks}

Unlike any standard fixed-point principle, Nielsen theory gives us additional information about the lower estimate of the fixed points number. It was originated by the Danish mathematician Jakob Nielsen in 1927 [N], who studied with this respect self-maps of compact surfaces. Later on, finite polyhedra were systematically treated by Franz Wecken in 1941-42 [W], who also gave an alternative definition of the Nielsen relations which we use below. The crucial step in this development was also accomplished by Andrzej Granas who defined a strictly related fixed-point index for arbitrary (i.e. also infinite-dimensional) ANRs in 1972 G].

Thus, a sufficiently general fixed-point principle, preserving the number of essential classes of fixed points under homotopy, could be formulated by U. K. Scholz $[\mathbf{S}$, Boju Jiang [Bo1], R. F. Brown [Br1], and some others (see the references in [AGJ1, [Bo1], Br1]). By sufficiency, we mean the appropriate form which was suitable for answering the question posed already by Jean Leray $[\mathrm{L}]$ at the first International Congress of Mathematicians held after World War II in Cambridge, Mass., in 1950. Namely, he suggested the problem of adapting the Nielsen theory to the needs of nonlinear analysis and, in particular, of its application to differential systems for obtaining multiplicity results (for more details see e.g. [Br3], [Br4]).

Since that time only several papers have been devoted to this problem (see e.g. $[\mathrm{BKM}], \overline{\mathrm{Br} 2}$, $[\mathrm{Br} 3],[\mathrm{Br} 4],[\mathrm{F} 1],[\mathrm{F} 2])$, but either additional parameters had to be

Received by the editors May 4, 1998 and, in revised form, November 6, 1998.

1991 Mathematics Subject Classification. Primary 34B15, 47H10.

Key words and phrases. Nielsen number, lower estimate of fixed points, multiplicity results, Carathéodory systems, nontrivial application. 
involved, as in the quoted papers by R. F. Brown and M. Fečkan, or a simple alternative approach could be used for the same goal, as in BKM.

Here we would like to present a nontrivial example of effective application of a noncompact version of the Nielsen fixed-point theory to differential equations without the above restrictions.

\section{TheOreticAl BACKGROUND}

In what follows, all topological spaces are assumed to be metric, and by a topology in function spaces (in particular, the Fréchet spaces $C\left(J, \Re^{n}\right)$ ) we mean the one of a uniform convergence on compact intervals. By a (metric) ANR-space $Y$ we understand, as usual, the one such that for any (metric) space $X$, its closed subset $S \subset X$ and a continuous mapping $f: S \rightarrow Y$, there exists an extension of $f$ onto some neighbourhood of $S$ in $X$.

Following $[\underline{S}]$, the basic notion of the Nielsen number will be defined for a socalled admissible class $A$ of self-maps $f: X \rightarrow X$, namely those satisfying:

(i) $f$ has a generalized Lefschetz number (for its definition and more details see e.g. [Br1], Bo1])

(ii) the set of fixed points, $\operatorname{Fix}(f)=\{\widehat{x} \in X: f(\widehat{x})=\widehat{x}\}$, is compact,

(iii) $X$ is a (metric) ANR-space.

Similarly, an admissible homotopy is a map $h: X \times[0,1] \rightarrow X$ such that $h_{t} \in$ $A$ and $H_{r, s} \in A$ for all $r, s, t \in[0,1]$, where $h_{t}(x)=h(x, t)$ and $H_{r, s}(x, t)=$ $[h(x,(1-t) r+t s),(1-t) r+t s]$, i.e. $\operatorname{Fix}(h)=\overline{\bigcup_{t \in[0,1]} \operatorname{Fix}\left(h_{t}\right)}$ is compact.

Definition $1(\underline{\underline{S}},[\mathrm{~W}])$. Given $f \in A$ and $x, y \in \operatorname{Fix}(f)$, we say that $x$ and $y$ are Nielsen-related (written $x \underset{N}{\sim} y$ ) if there exists a path $u:[0,1] \rightarrow X$ so that $u(0)=x$, $u(1)=y$ and $u, f(u)$ are homotopic keeping endpoints fixed.

One can readily check that the relation ". ${ }_{N}$. " is an equivalence. Furthermore, it is known (see $[\mathbf{S}]$ ) that each fixed-point class is open in $\operatorname{Fix}(f)$ and hence, in view of (ii), the number of such classes is finite. Therefore, we can give

Definition 2 ([Bo1], $[\mathbf{S}]$ ). If, for a Nielsen class $C \subset \operatorname{Fix}(f)$, we have $\operatorname{ind}(C, f) \neq$ 0 , i.e. if the associated fixed-point index in the sense of $G$ (see also [Bo1]) is nontrivial, then $C$ is called essential. The Nielsen number $N(f)$ is then defined to be the number of essential Nielsen classes.

Lemma 1 ([S] $)$. If $f \in A$ is admissible, then $f$ admits at least $N(f)$ fixed points, i.e. $N(f) \leq \# \operatorname{Fix}(f)$. Moreover, $N(f)$ is invariant under admissible homotopy $h: X \times[0,1] \rightarrow X$, i.e. if $h(0)=f$ and $h(1)=g$, then $N(f)=N(g)$.

The following lemma is essentially due to J. Jezierski [J].

Lemma 2 (reduction). Let $X$ and its closed subset $Y$ be ANR-spaces. Assume that $f: X \rightarrow X$ is a compact map, i.e. $\overline{f(X)}$ is compact, such that $f(X) \subset Y$. Denoting by $f^{\prime}: Y \rightarrow Y$ the restriction of $f$, we have

(i) $\operatorname{Fix}\left(f^{\prime}\right)=\operatorname{Fix}(f)$,

(ii) the Nielsen relations coincide,

(iii) $\operatorname{ind}\left(C, f^{\prime}\right)=\operatorname{ind}(C, f)$ for any Nielsen class $C \subset \operatorname{Fix}(f)$.

Thus, $N\left(f^{\prime}\right)=N(f)$. 
Proof. It is evident that $\operatorname{Fix}\left(f^{\prime}\right)=\operatorname{Fix}(f)$ and if $x, y$ are Nielsen-related in $\operatorname{Fix}\left(f^{\prime}\right)$, then so they are in $\operatorname{Fix}(f)$. To prove the converse, let us assume that $x, y \in \operatorname{Fix}(f)$ are Nielsen-related. This means that there is a path $u:[0,1] \rightarrow X$ satisfying

$$
H(t, 0)=u(t), \quad H(t, 1)=f \circ u(t), \quad H(0, s)=x, \quad H(1, s)=y
$$

with a suitable continuous mapping $H$. However, then $u^{\prime}(t)=f \circ u(t)$ is a path in $Y$ satisfying

$$
H^{\prime}(t, 0)=u^{\prime}(t), \quad H^{\prime}(t, 1)=f \circ u^{\prime}(t), \quad H^{\prime}(0, s)=f(x), \quad H^{\prime}(1, s)=f(y),
$$

where $H^{\prime}(t, s)=f \circ H(t, s)$, which already means that $x, y$ are Nielsen-related as the fixed points of $f^{\prime}: Y \rightarrow Y$.

Since the third property (iii) for the fixed-point indices is well known (see [Bo1, G]), the proof is complete.

Definition 3. We say that a mapping $T: Q \rightarrow S$ is retractible onto $Q$ if there is a retraction $r: P \rightarrow Q$, where $P$ is an open subset of $C\left(J, \Re^{n}\right)$ (i.e. a set of continuous maps from $J$ to $\Re^{n}$ ) containing $Q \cup S$, and $p \in P \backslash Q, r(p)=q$ implies that $p \neq T(q)$.

The main advantage of the above definition, already employed in [Br2], $\mathrm{Br} 3$, [Br4], states that $r \circ T: Q \rightarrow Q$ has a fixed point $\widehat{q} \in Q$ if and only if $\widehat{q}=T(\widehat{q})$.

The following statement, having for us a character of the method, represents only a special (single-valued) case of a more general (set-valued) version developed in AGJ2]. Let us note that it can also be derived as a combination of the results in $\underline{S}$, Theorems 1,2], [CFM, Theorem 1.1], Definition[3 and the fact that a neighbourhood retract in Fréchet spaces (in particular $C\left(J, \Re^{n}\right)$ ) is at the same time an ANR.

Theorem 1. Let $G: J \times \Re^{n} \times \Re^{n} \rightarrow \Re^{n}$ be a Carathéodory mapping, where $J$ is an arbitrary interval. Assume, furthermore, that there exists a (nonempty) closed connected subset $Q$ of $C\left(J, \Re^{n}\right)$ such that the problem

$$
\left\{\begin{array}{l}
X^{\prime}=G(t, X, q(t)), \\
X \in S
\end{array}\right.
$$

has, for every $q \in Q$, a unique solution $X(t)=T(q)$ with the property $\overline{T(Q)} \subset S$, where $S$ is a (nonempty) bounded subset of $C\left(J, \Re^{n}\right)$, and $T: Q \rightarrow S$ is retractible onto $Q$ with a retraction $r$ in the sense of Definition 3 .

At last, let there exist a locally Lebesgue-integrable function $\alpha: J \rightarrow \Re$ such that

$$
\mid G(t, X(t), q(t) \mid \leq \alpha(t) \text { a.e. in } J
$$

for any pair $(q, X) \in \Gamma_{T}$, where $\Gamma_{T}$ denotes the graph of $T$.

Then the Carathéodory system

$$
X^{\prime}=F(t, X)
$$

admits at least $N\left(\left.r\right|_{T(Q)} \circ T().\right)$ solutions belonging to $Q$, provided $G(t, c, c)=$ $F(t, c)$ takes place a.e. in $J$, for any $c \in \Re^{n}$.

A Carathéodory mapping $F: J \times \Re^{n} \rightarrow \Re^{n}$ means, as usual (cf. e.g. [F]), that:

(i) the mapping $F(t,$.$) is continuous for a.a. t \in J$,

(ii) the mapping $F(., X)$ is measurable for all $X \in \Re^{n}$.

Let us add that by a solution $X(t)$ we always understand a locally absolutely continuous function $X(t)$ satisfying the given system for a.a. $t \in J$. 


\section{NONTRIVIAL EXAMPLE}

Consider the Carathéodory system

$$
\begin{aligned}
& x^{\prime}+a x=e(t, x, y) y^{\frac{1}{m}}+g(t, x, y), \\
& y^{\prime}+b y=f(t, x, y) x^{\frac{1}{n}}+h(t, x, y),
\end{aligned}
$$

where $a, b$ are positive numbers and $m, n$ are odd integers with $\min (m, n) \geq 3$. Let suitable positive constants $E_{0}, F_{0}, G, H$ exist such that

$$
\begin{array}{cc}
|e(t, x, y)| \leq E_{0}, & |f(t, x, y)| \leq F_{0} \\
|g(t, x, y)| \leq G, & |h(t, x, y)| \leq H
\end{array}
$$

hold for a.a. $t \in(-\infty, \infty)$ and all $(x, y) \in \Re^{2}$.

Furthermore, assume the existence of positive constants $e_{0}, f_{0}, \delta_{1}, \delta_{2}$ such that

$$
0<e_{0} \leq e(t, x, y)
$$

for $x \geq-\delta_{1}, y \geq \delta_{2}$ and a.a. $t$ as well as for $x \leq \delta_{1}, y \leq-\delta_{2}$ and a.a $t$, jointly with

$$
0<f_{0} \leq f(t, x, y)
$$

for $x \geq \delta_{1}, y \leq \delta_{2}$ and a.a. $t$ as well as for $x \leq-\delta_{1}, y \geq-\delta_{2}$ and a.a. $t$.

Another possibility is that (2) holds for $x \leq \delta_{1}, y \geq \delta_{2}$ and a.a. $t$ as well as for $x \geq-\delta_{1}, y \leq-\delta_{2}$ and a.a. $t$ and that (3) holds at the same time for $x \geq \delta_{1}$, $y \geq-\bar{\delta}_{2}$ and a.a. $t$ as well as for $x \leq-\delta_{1}, y \leq \delta_{2}$ and a.a. $t$.

As a constraint $S$, consider at first the periodic boundary condition

$$
(x(0), y(0))=(x(\omega), y(\omega)) .
$$

More precisely, we take $S=Q=Q_{1} \cap Q_{2} \cap Q_{3}$, where

$$
\begin{aligned}
Q_{1}=\left\{q(t) \in C\left([0, \omega], \Re^{2}\right):\|q(t)\|=\max \left[\max _{t \in[0, \omega]}\left|q_{1}(t)\right|,\right.\right. \\
\left.\left.\max _{t \in[0, \omega]}\left|q_{2}(t)\right|\right] \leq R\right\}, \\
Q_{2}=\left\{q(t) \in C\left([0, \omega], \Re^{2}\right): \min _{t \in[0, \omega]}\left|q_{1}(t)\right| \geq \delta_{1}>0\right. \\
\left.\quad \text { or } \min _{t \in[0, \omega]}\left|q_{2}(t)\right| \geq \delta_{2}>0\right\}, \\
Q_{3}=\left\{q(t) \in C\left([0, \omega], \Re^{2}\right): q(0)=q(\omega)\right\}
\end{aligned}
$$

the constants $\delta_{1}, \delta_{2}, R$ will be specified below.

One can readily check that $Q$ is a nonempty connected subset of $C\left(\Re, \Re^{2}\right)$ and that, in order to verify $\overline{T(Q)} \subset S=Q$, it is sufficient to prove only $T(Q) \subset Q$, because $S=Q$ is closed. Moreover, $T(q)$ would be trivially retractible onto $Q$, when $T(Q) \subset Q$, because $Q$ is a neighbourhood retract of the Banach space $Q_{3}$, i.e. also an ANR-space.

Indeed. Defining

$$
U=\left\{q \in Q_{3}: \min _{t \in[0, \omega]}|q(t)|>0\right\},
$$


$U$ is obviously an open neighbourhood of $Q$ in $Q_{3}$. The related retraction $r: U \rightarrow Q$, for every $t \in[0, \omega]$, takes the form

$$
r(q)(t)= \begin{cases}\frac{q(t)}{|q(t)|} R \text { for }|q(t)|>R, \\ \left(\frac{q_{1}(t)}{\left|q_{1}(t)\right|} \delta_{1}, \frac{q_{2}(t)}{\left|q_{1}(t)\right|} \delta_{1}\right) & \text { for }\left|q_{2}(t)\right|<\frac{\delta_{2}}{\delta_{1}}\left|q_{1}(t)\right|<\delta_{2}, \\ \left(\frac{q_{1}(t)}{\left|q_{1}(t)\right|} \delta_{1}, \frac{q_{2}(t)}{\left|q_{2}(t)\right|} \delta_{2}\right) & \text { for } 0<\left|q_{2}(t)\right|<\frac{\delta_{2}}{\delta_{1}}\left|q_{1}(t)\right|<\delta_{2}, \\ \left(\frac{q_{1}(t)}{\left|q_{2}(t)\right|} \delta_{2}, \frac{q_{2}(t)}{\left|q_{2}(t)\right|} \delta_{2}\right) & \text { for } \delta_{2}>\left|q_{2}(t)\right|>\frac{\delta_{2}}{\delta_{1}}\left|q_{1}(t)\right|, \\ q(t), \quad \text { otherwise. } & \end{cases}
$$

Besides (1), consider still its embedding into

$$
\begin{aligned}
& x^{\prime}+a x=\left[(1-\mu) e_{0}+\mu e(t, x, y)\right] y^{\frac{1}{m}}+\mu g(t, x, y), \\
& y^{\prime}+b y=\left[(1-\mu) f_{0}+\mu f(t, x, y)\right] x^{\frac{1}{n}}+\mu h(t, x, y),
\end{aligned}
$$

where $\mu \in[0,1]$. Thus, (5) reduces to (1) for $\mu=1$.

The associated linearized system to (5) takes for $\mu \in[0,1]$ the form

$$
\begin{aligned}
& x^{\prime}+a x=\left[(1-\mu) e_{0}+\mu e_{t}\right] q_{2}(t)^{\frac{1}{m}}+\mu g_{t}, \\
& y^{\prime}+b y=\left[(1-\mu) f_{0}+\mu f_{t}\right] q_{1}(t)^{\frac{1}{n}}+\mu h_{t},
\end{aligned}
$$

where we use for simplicity the notation

$$
\begin{aligned}
& e_{t}:=e\left(t, q_{1}(t), q_{2}(t)\right), \quad f_{t}:=f\left(t, q_{1}(t), q_{2}(t)\right), \\
& g_{t}:=g\left(t, q_{1}(t), q_{2}(t)\right), \quad h_{t}:=h\left(t, q_{1}(t), q_{2}(t)\right) .
\end{aligned}
$$

It is well known that problem (6)-(4) has, for each $q(t) \in Q$, a unique solution $X(t)=(x(t), y(t)):=T_{\mu}(q)$, namely

$$
T_{\mu}(q)=\left\{\begin{array}{l}
x(t)=\int_{0}^{\omega} G_{1}(t, s)\left[\left((1-\mu) e_{0}+\mu e_{s}\right) q_{2}(s)^{\frac{1}{m}}+\mu g_{s}\right] d s, \\
y(t)=\int_{0}^{\omega} G_{2}(t, s)\left[\left((1-\mu) f_{0}+\mu f_{s}\right) q_{1}(s)^{\frac{1}{n}}+\mu h_{s}\right] d s,
\end{array}\right.
$$

where

$$
\begin{aligned}
& G_{1}(t, s)=\left\{\begin{array}{l}
\frac{\mathrm{e}^{-a(t-s+\omega)}}{1-\mathrm{e}^{-a \omega}} \text { for } 0 \leq t \leq s \leq \omega, \\
\frac{\mathrm{e}^{-a(t-s)}}{1-\mathrm{e}^{-a \omega}} \text { for } 0 \leq s \leq t \leq \omega,
\end{array}\right. \\
& G_{2}(t, s)=\left\{\begin{array}{l}
\frac{\mathrm{e}^{-b(t-s+\omega)}}{1-\mathrm{e}^{-b \omega}} \text { for } 0 \leq t \leq s \leq \omega, \\
\frac{\mathrm{e}^{-b(t-s)}}{1-\mathrm{e}^{-b \omega}} \text { for } 0 \leq s \leq t \leq \omega .
\end{array}\right.
\end{aligned}
$$

Since $X(0)=X(\omega)$, i.e. $T_{\mu}(Q) \subset Q_{3}$, our first goal is to show that $Q$ is invariant under $T_{\mu}(q)$, i.e. $T_{\mu}(Q) \subset Q$, for each $\mu \in[0,1]$. Hence, we should prove that $T_{\mu}(Q) \subset Q_{1}$ as well as $T_{\mu}(Q) \subset Q_{2}$.

Let us consider the first inclusion. In view of

$$
\min _{t, s \in[0, \omega]} G_{1}(t, s) \geq \frac{\mathrm{e}^{-a \omega}}{1-\mathrm{e}^{-a \omega}}>0
$$


and

$$
\min _{t, s \in[0, \omega]} G_{2}(t, s) \geq \frac{\mathrm{e}^{-b \omega}}{1-\mathrm{e}^{-b \omega}}>0,
$$

we obtain for the above solution $X(t)$ that

$$
\begin{aligned}
& \max _{t \in[0, \omega]}|x(t)| \leq \max _{t \in[0, \omega]} \int_{0}^{\omega}\left|G_{1}(t, s)\right|\left|\left[(1-\mu) e_{0}+\mu e_{s}\right] q_{2}(s)^{\frac{1}{m}}+\mu g_{s}\right| d s \\
& \leq\left[\left(e_{0}+E_{0}\right) R^{\frac{1}{m}}+G\right] \max _{t \in[0, \omega]} \int_{0}^{\omega} G_{1}(t, s) d s=\frac{1}{a}\left[\left(e_{0}+E_{0}\right) R^{\frac{1}{m}}+G\right]
\end{aligned}
$$

and

$$
\begin{aligned}
& \max _{t \in[0, \omega]}|y(t)| \leq \max _{t \in[0, \omega]} \int_{0}^{\omega}\left|G_{2}(t, s)\right|\left|\left[(1-\mu) f_{0}+\mu f_{s}\right] q_{1}(s)^{\frac{1}{n}}+\mu h_{s}\right| d s \\
& \leq\left[\left(f_{0}+F_{0}\right) R^{\frac{1}{n}}+H\right] \max _{t \in[0, \omega]} \int_{0}^{\omega} G_{2}(t, s) d s=\frac{1}{b}\left[\left(f_{0}+F_{0}\right) R^{\frac{1}{n}}+H\right] .
\end{aligned}
$$

Because of

$$
\begin{gathered}
\|X(t)\|=\max \left[\max _{t \in[0, \omega]}|x(t)|, \max _{t \in[0, \omega]}|y(t)|\right] \\
\leq \max \left\{\frac{1}{a}\left[\left(e_{0}+E_{0}\right) R^{\frac{1}{m}}+G\right], \frac{1}{b}\left[\left(f_{0}+F_{0}\right) R^{\frac{1}{n}}+H\right]\right\},
\end{gathered}
$$

a sufficiently big constant $R$ certainly exists such that $\|X(t)\| \leq R$, i.e. $T_{\mu}(Q) \subset Q_{1}$, independently of $\mu \in[0,1]$.

For the inclusion $T_{\mu}(Q) \subset Q_{2}$, we proceed quite analogously. Assuming that $q(t) \in Q_{2}$, we have either

$$
\min _{t \in[0, \omega]}\left|q_{1}(t)\right| \geq \delta_{1}>0 \text { or } \min _{t \in[0, \omega]}\left|q_{2}(t)\right| \geq \delta_{2}>0 .
$$

Therefore, we obtain for the above solution $X(t)$ that

$$
\begin{aligned}
& \min _{t \in[0, \omega]}|x(t)|=\min _{t \in[0, \omega]} \int_{0}^{\omega}\left|G_{1}(t, s)\right|\left|\left[(1-\mu) e_{0}+\mu e_{s}\right] q_{2}(s)^{\frac{1}{m}}+\mu g_{s}\right| d s \\
\geq & \left|e_{0} \delta_{2}^{\frac{1}{m}}-G\right| \int_{0}^{\omega} G_{1}(t, s) d s=\frac{1}{a}\left|e_{0} \delta_{2}^{\frac{1}{m}}-G\right|>0, \text { provided } G<e_{0} \delta_{2}^{\frac{1}{m}},
\end{aligned}
$$

for $q_{1} \geq-\delta_{1}, q_{2} \geq \delta_{2}$ as well as for $q_{1} \leq \delta_{1}, q_{2} \leq-\delta_{2}$ (or another alternative, as above), or

$$
\begin{aligned}
& \min _{t \in[0, \omega]}|y(t)|=\min _{t \in[0, \omega]} \int_{0}^{\omega}\left|G_{2}(t, s)\right|\left|\left[(1-\mu) f_{0}+\mu f_{s}\right] q_{1}(s)^{\frac{1}{n}}+\mu h_{s}\right| d s \\
\geq & \left|f_{0} \delta_{1}^{\frac{1}{n}}-H\right| \int_{0}^{\omega} G_{2}(t, s) d s=\frac{1}{b}\left|f_{0} \delta_{1}^{\frac{1}{n}}-H\right|>0, \text { provided } H<f_{0} \delta_{1}^{\frac{1}{n}},
\end{aligned}
$$

for $q_{1} \geq \delta_{1}, q_{2} \leq \delta_{2}$ as well as for $q_{1} \leq-\delta_{1}, q_{2} \geq-\delta_{2}$ (or another alternative, as above).

So, in order to prove that $X(t) \in Q_{2}$, we need to simultaneously fulfill the following inequalities:

$$
\left\{\begin{array}{l}
\frac{1}{a}\left|e_{0} \delta_{2}^{\frac{1}{m}}-G\right| \geq \delta_{1}>\left(\frac{H}{f_{0}}\right)^{n} \\
\frac{1}{b}\left|f_{0} \delta_{1}^{\frac{1}{n}}-H\right| \geq \delta_{2}>\left(\frac{G}{e_{0}}\right)^{m} .
\end{array}\right.
$$


Let us observe that the amplitudes of the functions $g, h$ must be sufficiently small. On the other hand, if $e_{0}$ and $f_{0}$ are sufficiently large (for fixed quantities $a, b, G$, $H$ ), then we can easily find $\delta_{1}, \delta_{2}$ satisfying (7).

After all, if there exist constants $\delta_{1}, \delta_{2}$ obeying (7), then we arrive at $X(t) \in Q_{2}$, i.e. $T_{\mu}(Q) \subset Q_{2}$, independently of $\mu \in[0,1]$. This already means that $T_{\mu}(Q) \subset Q$, independently of $\mu \in[0, \omega]$, as required.

Since the operators

$$
\left(T_{\mu}, \mu\right): Q \times[0,1] \rightarrow Q \times[0,1] \text { and }\left(T^{\nu}, \nu\right): Q \times[0,1] \rightarrow Q \times[0,1] \text { (see below) }
$$

can be easily verified to be completely continuous with closed fixed-point sets, all the associated homotopies are admissible in the sense of the above definition.

Now, since all the assumptions of Theorem 1 are satisfied, problem (5)-(4) possesses at least $N\left(T_{\mu}().\right)$ solutions belonging to $Q$, for every $\mu \in[0,1]$. In particular, problem (1)-(4) has $N\left(T_{1}().\right)$ solutions, but according to Lemma 1. $N\left(T_{1},().\right)=N\left(T_{0}().\right)$. So, it remains to compute the Nielsen number $N\left(T_{0}().\right)$ for the operator $T_{0}: Q \rightarrow Q$, where

$$
T_{0}(q)=\left\{\begin{array}{l}
e_{0} \int_{0}^{\omega} G_{1}(t, s) q_{2}(s)^{\frac{1}{m}} d s \\
f_{0} \int_{0}^{\omega} G_{2}(t, s) q_{1}(s)^{\frac{1}{n}} d s .
\end{array}\right.
$$

Hence, besides (8), consider still its embedding into one-parameter family of operators

$$
T^{\nu}(q)=\nu T_{0}(q)+(1-\nu) r \circ T_{0}(q), \quad T^{\nu}: Q \rightarrow Q, \quad \nu \in[0,1],
$$

where $r(q):=\left(r\left(q_{1}\right), r\left(q_{2}\right)\right)$ and

$$
r\left(q_{i}\right)=q_{i}(0) \text { for } i=1,2 .
$$

One can readily check that $r: Q \rightarrow Q \cap \Re^{2}$ is a retraction and $T_{0}(\bar{q}): Q \cap \Re^{2} \rightarrow Q$ is retractible onto $Q \cap \Re^{2}$ with the retraction $r$ in the sense of Definition 3. Thus, $r \circ T_{0}(\bar{q}): Q \cap \Re^{2} \rightarrow Q \cap \Re^{2}$ has a fixed point $\widehat{q} \in Q \cap \Re^{2}$ if and only if $\widehat{q}=T_{0}(\widehat{q})$. Moreover, $r \circ T_{0}(q): Q \rightarrow Q \cap \Re^{2}$ has evidently a fixed point $\widehat{q} \in Q \cap \Re^{2}$ if and only if $\widehat{q}=T_{0}(\widehat{q})$. So, the investigation of fixed points for $T^{0}(q)=r \circ T_{0}(q)$ turns out to be equivalent with the one for $T^{0}(\bar{q}): Q \cap \Re^{2} \rightarrow Q \cap \Re^{2}$.

Since, in view of Lemma 1, we have

$$
N\left(T_{1}(.)\right)=N\left(T_{0}(.)\right)=N\left(T^{1}(.)\right)=N\left(T^{0}(.)\right)
$$

where

$$
T^{0}(q)=\left(\frac{e_{0} \mathrm{e}^{-a \omega}}{1-\mathrm{e}^{-a \omega}} \int_{0}^{\omega} \mathrm{e}^{a s} q_{2}(s)^{\frac{1}{m}} d s, \frac{f_{0} \mathrm{e}^{-b \omega}}{1-\mathrm{e}^{-b \omega}} \int_{0}^{\omega} \mathrm{e}^{b s} q_{1}(s)^{\frac{1}{n}} d s\right)
$$

and

$$
T^{0}(\bar{q})=\left(\frac{e_{0}}{a} \bar{q}_{2} \frac{1}{m}, \frac{f_{0}}{b} \bar{q}_{1} \frac{1}{n}\right) \quad \text { for } \bar{q}=\left(\overline{q_{1}}, \overline{q_{2}}\right)=\left(q_{1}(0), q_{2}(0)\right) \in Q \cap \Re^{2},
$$

it remains to estimate $N\left(T^{0}().\right)$. It will be useful to do it by passing to a simpler finite-dimensional analogy, namely by the direct computation of fixed points of the operator

$$
T^{0}(\bar{q}): Q \cap \Re^{2} \rightarrow Q \cap \Re^{2},
$$

belonging to different Nielsen classes. 
There are two fixed points $\widehat{q}_{+}=\left(\widehat{q}_{1}, \widehat{q}_{2}\right)$ and $\widehat{q}_{-}=\left(-\widehat{q}_{1},-\widehat{q}_{2}\right)$ in $Q \cap \Re^{2}$, where

$$
\begin{aligned}
& \widehat{q}_{1}=\left(\frac{e_{0}}{a}\right)^{\frac{m n}{m n-1}}\left(\frac{f_{0}}{b}\right)^{\frac{1}{m n-1}}, \\
& \widehat{q}_{2}=\left(\frac{e_{0}}{a}\right)^{\frac{m}{m n-1}}\left(\frac{f_{0}}{b}\right)^{\frac{m n}{m n-1}} .
\end{aligned}
$$

These fixed points belong to different Nielsen classes, because any path $u$ connecting them in $Q \cap \Re^{2}$ and its image $T^{0}(u)$ are not homotopic in the space $Q \cap \Re^{2}$.

Thus, according to Definition 2, $N\left(T^{0}(\bar{q})\right)=2$. By means of Lemma 2, we have moreover $N\left(T_{1}().\right)=N\left(T^{0}().\right)=N\left(T^{0}(\bar{q})\right)=2$ and so, according to Theorem 1 system (1) admits at least two solutions belonging to $Q$, provided suitable positive constants $\delta_{1}, \delta_{2}$ exist satisfying (5).

In fact, system (11) possesses at least three solutions satisfying (4), when the sharp inequalities appear in (7), by which the lower boundary of $Q$ becomes fixedpoint free. Indeed. Since $\Lambda\left(T_{1}(), Q.\right)=\Lambda\left(T^{0}(), Q.\right)=\Lambda\left(T^{0}(\bar{q}), Q \cap \Re^{2}\right)$ holds for the generalized Lefschetz numbers (see e.g. [Br1]) and one can easily check that $\Lambda\left(T^{0}(\bar{q}), Q \cap \Re^{2}\right)=2$, we obtain that $\Lambda\left(T_{1}(), Q.\right)=2$. Furthermore, since for the self-map $T_{1}($.$) on convex set Q_{1} \cap Q_{3}$ we have $\Lambda\left(T_{1}(),. Q_{1} \cap Q_{3}\right)=1$ (see e.g. [Br1]), it follows from the additivity, excision and existence properties of the fixed point index (see [Bo1, G]) that the mapping $T_{1}($.$) has the third fixed point in$ $Q_{1} \cap Q_{3} \backslash Q$, representing a solution of problem (1) -(4) and belonging to $Q_{1} \backslash Q$.

As we can see, problem (1)-(4) admits at least two solutions in $Q_{1} \cap Q_{3}$ for an arbitrary $\omega>0$. Furthermore, because of rescaling (1), when replacing $t$ by $t+\frac{\omega}{2}$, there are also two solutions of (11) satisfying $X\left(-\frac{\omega}{2}\right)=X\left(\frac{\omega}{2}\right)$, for an arbitrary $\omega>0$, and belonging to $Q_{1}$. Therefore, according to the intuitively clear Lemma 2.8.1 in [K] and by the obvious geometrical reasons related to the appropriate subdomains of $Q$, system (11) possesses at least two bounded solutions in $Q_{1}$.

Of course, because of replacing $t$ by $(-t)$ the same result holds for (1) with negative constants as well.

Finally, consider the system of inclusions

$$
\begin{aligned}
& x^{\prime}+a x \in e(t, x, y) y^{\frac{1}{m}}+g(t, x, y), \\
& y^{\prime}+b y \in f(t, x, y) x^{\frac{1}{n}}+h(t, x, y),
\end{aligned}
$$

where $a, b, m, n$ are same, but $e, f, g, h$ are this time multifunctions, which are product-measurable for all $(t, x, y) \in \Re^{3}$, lower-semicontinuous in $(x, y)$ for a.a. $t \in(-\infty, \infty)$, and with the same estimates as above.

Since each mapping $e, f, g, h$ has, under our regularity assumptions, a Carathéodory selector (see e.g. $[\mathrm{R}$ and the references therein), the same must be also true for (9).

Summing up the above conclusions, we can give finally

Theorem 2. Let suitable positive constants $\delta_{1}, \delta_{2}$ exist such that the inequalities

$$
\begin{aligned}
& \frac{1}{|a|}\left|e_{0} \delta_{2}^{\frac{1}{m}}-G\right| \geq \delta_{1}>\left(\frac{H}{f_{0}}\right)^{n}, \\
& \frac{1}{|b|}\left|f_{0} \delta_{1}^{\frac{1}{n}}-H\right| \geq \delta_{2}>\left(\frac{G}{e_{0}}\right)^{m}
\end{aligned}
$$


are satisfied for constants $e_{0}, f_{0}, G, H$ estimating the multifunctions $e, f, g, h$ as above, for constants $a, b$ with $a b>0$ and for odd integers $m, n$ with $\min (m, n) \geq 3$. Then system (9) admits at least two entirely bounded solutions.

If, in particular, the sharp inequalities appear in (10), then system (9) admits at least three $\omega$-periodic solutions, provided the multifunctions $e, f, g, h$ are $\omega$-periodic in $t$.

As a direct consequence of the existence of three harmonics, system (1) with $\omega$-periodic in $t$ and right-hand sides satisfying suitable regularity assumptions possesses "generically", in view of T. Matsuoka's results in [M1], M2] (cf. also [Bo2], [HJ]), infinitely many periodic (subharmonic) solutions, provided the same assumptions as above are satisfied.

The "genericity" is understood in the sense of Artin's braid group theory, i.e. when the only exceptional braids representing periodic solutions are certain simplest ones (for more details see [Bo2]).

So, we can conclude by

Corollary. Assume, in particular, that $\left[e(t, x, y) y^{\frac{1}{m}}+g(t, x, y)\right]$ and $\left[f(t, x, y) x^{\frac{1}{n}}+\right.$ $h(t, x, y)]$ are Carathéodory-functions on $\Re \times \Re^{2}$ such that a uniqueness property for Cauchy problems is guaranteed and that $e(t, x, y) \equiv e(t+\omega, x, y), f(t, x, y) \equiv$ $f(t+\omega, x, y), g(t, x, y) \equiv g(t+\omega, x, y), h(t, x, y) \equiv h(t+\omega, x, y)$. Let positive constants $\delta_{1}, \delta_{2}$ exist such that the sharp inequalities take place in (10) for constants $e_{0}, f_{0}, G, H$ estimating the functions $e, f, g, h$ as above, for constants $a, b$ with $a b>0$ and for odd integers $m, n$ with $\min (m, n) \geq 3$. Then system (1) has generically infinitely many periodic (subharmonic) solutions.

Example. Consider the system

$$
\begin{aligned}
& x^{\prime}+x=e(x, y) y^{\frac{1}{3}}+\cos 2 \pi t, \\
& y^{\prime}+y=f(x, y) x^{\frac{1}{5}}+\cos 2 \pi t
\end{aligned}
$$

where $\left(\delta_{1}=10^{-4}, \delta_{2}=10^{-2}, R=100\right)$

$e(x, y)=\left\{\begin{array}{l}10 \text { for }(x, y) \in \Re^{2} \backslash\left\{\left(-R<x<-\delta_{1} \wedge \delta_{2}<y<R\right)\right. \\ \left.\quad \vee\left(\delta_{1}<x<R \wedge-R<y<-\delta_{2}\right) \vee\left(|x|<\delta_{1} \wedge|y|<\delta_{2}\right)\right\}, \\ 0 \text { for }(x, y) \in\left\{\left(\frac{-R-\delta_{1}}{2}, \frac{R+\delta_{2}}{2}\right),\left(\frac{R+\delta_{1}}{2}, \frac{-R-\delta_{2}}{2}\right)\right\}, \\ \text { any extension with }|e(x, y)| \leq 10 \text { such that } e(x, y) y^{\frac{1}{3}} \\ \text { is locally lipschitzian, otherwise, }\end{array}\right.$

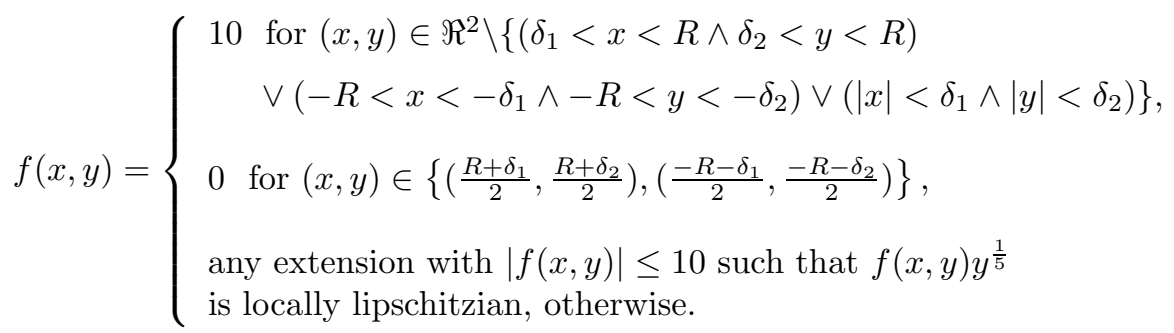


According to Theorem 2, system (11) admits three 1-periodic solutions and so, in view of the Corollary, it might have infinitely many $p$-periodic (subharmonic) solutions, $p \in \mathbf{N}$.

\section{REFERENCES}

[AGJ1] J. Andres, L. Górniewicz and J. Jezierski: A generalized Nielsen number and multiplicity results for differential inclusions. To appear in Topol. Appl. 12 (1999).

[AGJ2] J. Andres, L. Górniewicz And J. Jezierski: Noncompact version of the multivalued Nielsen theory and its application to differential inclusions. Lecture Notes of the Schauder Center 2: "Differential Inclusions and Optimal Control" (Proceedings of the Banach Center Workshop held in Warsaw, September 27-October 3, 1997), (J. Andres, L. Górniewicz and P. Nistri, eds.), 33-50.

[BKM] A. Yu. Borisovich, Z. Kucharski and W. Marzantowicz: A multiplicity result for a system of real integral equations by use of the Nielsen number. Preprint (1997).

[Bo1] BoJU JIAng: Nielsen Fixed Point Theory. Contemp. Math. 14, AMS, Providence, RI, 1983. MR 84f:55002

[Bo2] BoJU JiAng: Nielsen Theory for periodic orbits and applications to dynamical systems. In: "Nielsen Theory and Dynamical Systems" (C. K. McCord, ed.), Contemp. Math. 152, Amer. Math. Soc., Providence, R.I., 1993, 183-202. MR 94k:55002

[Br1] R. F. Brown: The Lefschetz Fixed Point Theorem. Scott, Foresman and Comp., Glenview, Illinois, 1971. MR 44:1023

[Br2] R. F. Brown: Multiple solutions to parametrized nonlinear differential systems from Nielsen fixed point theory. In: Nonlinear Analysis (ed. by Th. M. Rassias), World Scientific Publ. Co., Singapore (1987), 89-98. MR 89e:47086

[Br3] R. F. Brown: Topological identification of multiple solutions to parametrized nonlinear equations. Pacific J. Math. 131,1 (1988), 51-69. [MR 89e:47087

[Br4] R. F. Brown: Nielsen fixed point theory and parametrized differential equations. Contemp. Math. 72, AMS, Providence, RI, 1988, 33-45. MR 89c:00036

[CFM] M. CECChI, M. Furi And M. MARINi: About the solvability of ordinary differential equations with asymptotic boundary conditions. Boll. U.M.I. Ser. VI, 4-C, 1 (1985), 329-345. MR 86k:34009

[F] A. F. Filippov: Differential Equations with Discontinuous Right Hand Sides. Kluwer, Dordrecht, 1988. MR 90i:34002

[F1] M. FeČKAn: Nielsen fixed point theory and nonlinear equations. J. Diff. Eqns. 106, 2 (1993), 312-331. MR 94k:47093

[F2] M. FEČKAN: Multiple periodic solutions of small vector fields on differentiable manifolds. J. Diff. Eqns. 113, 1 (1994), 189-200. MR 95h:34058

[G] A. Granas: The Leray-Schauder index and the fixed point theory for arbitrary ANRs. Bull. Soc. Math. France 100 (1972), 209-228. MR 46:8213

[HJ] H.-H. Huang And B.--J. Jiang: Braids and periodic solutions. In: Springer LNM 1411, "Topological Fixed Point Theory and Applications" (B. Jiang, ed.), Springer, Berlin, 1989, 107-123. MR 91g:58227

[J] J. Jezierski: Private communication.

[K] M. A. KRASnosel'SkII: The Operator of Translation along the Trajectories of Differential Equations. TMM 19, Amer. Math. Soc., Providence, R.I., 1968. MR 36:6688

[L] J. LeraY: La theorie des points fixes et ses applications en analyse. In: Proc. International Congres of Math., 1950, Vol. 2, Amer. Math. Soc., 1952. MR 13:859a

[M1] T. MATSUOKA: The number and linking of periodic solutions of periodic systems. Invent. Math. 70 (1983), 319-340. MR 84g:58093

[M2] T. MATSUOKA: Wave form in the dynamical study of ordinary differential equations. Japan J. Appl. Math. 1, 2 (1984), 417-434. MR 87i:34044

[N] J. NiElSEN: Untersuchungen zur Topologie der geschlossenen zweiseitigen Flächen. Acta Math. 50 (1927), 189-358.

[R] L. Rybinski: On Carathéodory type selections. Fund. Math. 125 (1985), 187-193. MR 87k:28012 
[S] K. Scholz: The Nielsen fixed point theory for non-compact spaces. Rocky Mount. J. Math. 4 (1974), 81-87.

[W] F. Wecken: Fixpunktklassen. I, Math. Ann. 117 (1941), 659-671; II, 118 (1942), 216234; III, 118 (1942), 544-577. MR 3:140b MR 5:275a MR 5:275b

Department of Mathematical Analysis, Faculty of Science, Palacký University, Tomkova 40, 77900 Olomouc-HejČín, Czech Republic

E-mail address: andres@risc.upol.cz 